\title{
Evidence for a Mechanism that Involves Secondary Electron Capture in the Liquid Secondary Ionization Mass Spectrometry Beam-Induced Dehalogenation of Organic Compounds
}

\author{
R. Théberge and M. J. Bertrand \\ Regional Center for Mass Spectrometry, Department of Chemistry, University of Montreal, Montreal, Canada
}

\begin{abstract}
The effect of the analyte electron affinity on the liquid secondary ionization mass spectrometry beam-induced dehalogenation of simple bromoaromatic compounds in a glycerol matrix was investigated. The results show a definite trend of decreasing dehalogenation with increasing analyte electron affinity. At high analyte electron affinity $(\geq 1.0 \mathrm{eV})$, no dehalogenation was observed. These results are consistent with electrochemical and pulse radiolysis studies where one electron reduction was shown to be responsible for dehalogenation. A chloroaromatic compound with high electron affinity, 4-(4-chloro-benzoyl)pyridine, exhibited reduction by hydrogen addition but not dehalogenation. The radiation chemistry of alcohols was used to elaborate a scheme of the reactive species generated in the glycerol matrix by kiloelectronvolt particle bombardment. The possible role of those species in reduction processes such as dehalogenation was evaluated. The observation that dehalogenation decreases with analyte electron affinity is mechanistically consistent with the proposition that secondary electron production is an intrinsic part of the bombardment process. (C) 1996 American Society for Mass Spectrometry (J Am Soc Mass Spectrom 1996, 7, 1109-1115)
\end{abstract}

$\mathrm{D}$ espite the widespread applications of fast-atom bombardment-liquid secondary ionization mass spectrometry (FAB-LSIMS) and the concomitant broadening of the scope of mass spectrometry that ensued from the introduction of the technique [1], basic fundamental processes related to the generation of analytically relevant ions have remained nebulous. Although the nature of the ionization process has generated considerable attention, speculation, and study, mass spectrometrists have been concerned by the possibility that artifacts originate from beam-induced reactions of the sample $[2,3]$. This phenomenon can complicate spectrum interpretation significantly or lead to erroneous conclusions as to the nature of the analyte.

One such type of beam-induced reaction involves the substitution of a halogen by a hydrogen, which results in the generation of a dehalogenated artifact [4-11]. Dehalogenated artifacts also have been observed in ${ }^{252} \mathrm{Cf}$ desorption [12] and thermospray [13, 14]. The FAB-LSIMS dehalogenation process has been

Address reprint requests to Dr. M. J. Bertrand, Regional Center for Mass Spectrometry, Department of Chemistry, University of Montreal, P.O. Box 6128, Montreal, Canada H3C 317. the subject of several studies [4-11]. Sethi et al. [6] invoked the resemblance of beam-induced dehalogenation with that of radiolysis studies where reactions are initiated by the solvated electron. Williams et al. [15] suggested a mechanism based on the production of electrons due to the interaction of the primary beam with the sample to account for the occurrence of dehalogenation in the FAB-LSIMS spectra of organic compounds. The complete or substantial inhibition of dehalogenation observed when electron-scavenging matrices were used was interpreted as evidence for an electron-based mechanism [9-11].

To further our understanding of the beam-induced dehalogenation of haloaromatics [10, 11], we have studied the effect of analyte electron affinity on the extent of dehalogenation observed in the liquid secondary ionization mass spectrometry (LSIMS) spectra. This line of investigation is important in light of the oft-repeated suggestion that FAB-LSIMS beaminduced reductions involve the availability of low-lying unoccupied molecular orbitals that can capture electrons produced by the interaction of the beam with the sample and initiate further reactions such as dehalogenation. The analyte electron affinity is a measure of the availability of such low-lying unoccupied orbitals 
as defined by the lowest unoccupied molecular orbital (LUMO). This investigation also provides the opportunity to verify the observation of Kelley and Musser [9] over a much larger range of electron affinities with simpler compounds. These authors noted a decrease in dehalogenation with increased analyte electron affinity. In that study, the range of analyte electron affinities was very small $(0.27 \mathrm{eV})$. The existence and direction of such an analyte electron affinity effect may allow for some mechanistic inferences to be made to implicate beam-generated electrons as initiating agents in the dehalogenation process. In turn, such mechanistic inferences may be substantiated by using results obtained with pulse radiolysis and electrochemical techniques where the compounds studied are similar and electrons are the initiating reagents in the dehalogenation process.

\section{Experimental}

Mass spectral data were obtained by using a VG Autospec-Q hybrid spectrometer (VG Analytical, Manchester, UK) equipped with a cesium ion gun. The accelerating voltage was $8 \mathrm{kV}$ and the mass resolution 1000. Magnet scans (5 s/decade) were used over a mass range of $50-1000 \mu$. The cesium ion beam energy and beam current were held constant at $22 \mathrm{keV}$ and 2 $\mu \mathrm{A}$, respectively. A square probe tip of area $7 \mathrm{~mm}^{2}$ was used to ensure uniform irradiation of the sample. The structures of the compounds used in this study are shown in Figure 1. Compound 5 was prepared by using the EDC (1-[3-dimethylaminopropyl]-3-ethylcarbodiimide hydrochloride) coupling of 2-bromo-2nitrobenzoic acid and 2-aminoethylpiperidine to yield the amide. This is a typical use of carbodiimide reagents to couple carboxylic acids with amines to obtain an amide [16]. The product was purified by using flash chromatography (ethyl acetate and $0.2 \%$ triethyl amine). Compound 4 was obtained by the reaction of $4^{\prime}$-bromo-4-chlorobutyrophenone with triethyl amine. The methyl ester of 4-bromo-phenylalanine was prepared from the zwitterionic form of the amino acid by reaction with thionyl chloride in methanol. All other compounds were obtained from Aldrich Chemical Co. (Milwaukee, WI) and used without further purification. The percent dehalogenation values are average values obtained from the first $2 \mathrm{~min}$ of analysis. The concentration of the analytes was 0.04 $M$. The only matrices used in this study were NBA (3-nitrobenzyl alcohol) and glycerol. Analysis was performed immediately following mixture of the analyte with the matrix.

The modified neglect of differential overlap MNDO calculations for compounds 1-5 were performed on a Silicon Graphics LISA workstation that used MOPAC (version 6.0). Because the electron affinities of compounds 1-5 were not known, they were calculated from the lowest unoccupied molecular orbital (LUMO)

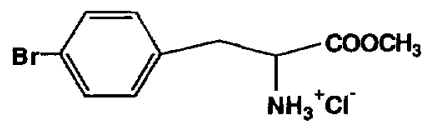

(1)
(3)<smiles>NC(=O)c1ccc(Br)cc1</smiles>

(4)<smiles>NC(=O)c1ccc(Br)cc1</smiles><smiles>Nc1ccc(Br)c2ccccc12</smiles>

(2)
(5)

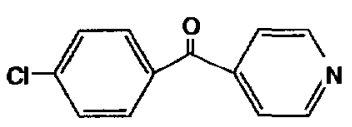

(6)

Figure 1. Structure of the compounds used for the study.

in a manner similar to that described by Laramée et al. [17].

\section{Results and Discussion}

To study the effect of analyte electron affinity, simple substituted bromoaromatics (Figure 1) were used. Such compounds were used to ensure a wide range of electron affinities and a propensity to undergo dehalogenation. The percent dehalogenation was defined as

$$
\% \text { Dehalogenation }=\frac{100\left(\mathrm{M}_{\mathrm{H}}+\mathrm{H}\right)^{+}}{\left(\mathrm{M}_{\mathrm{H}}+\mathrm{H}\right)^{+}+\left(\mathrm{M}_{\mathrm{X}}+\mathrm{H}\right)^{+}}
$$

where $\mathrm{M}_{\mathrm{X}} \mathrm{H}^{+}$and $\mathrm{M}_{\mathrm{H}} \mathrm{H}^{+}$are the relative abundances of the intact protonated molecule and dehalogenated species, respectively. Because dehalogenation occurs most readily when glycerol is the matrix [9-11], all percent dehalogenation values used in the correlation with analyte electron affinity were obtained with glycerol as the matrix. Electron affinities (EAs) for the compounds of Figure 1 are not readily available; however, they may be expected to correlate with LLMO values, which can be calculated readily by using the MOPAC program. To quantatively assess the correlation, LUMOs were calculated for the three compounds of known electron affinity [18] listed in Table 1. The linear regression of these EA and LUMO values gives 
Table 1. Electron affinities [18] and calculated LUMO values (estimated by using MOPAC) used for the elaboration of the calibration graph

\begin{tabular}{lcc} 
Compound & $\begin{array}{c}\text { Electron } \\
\text { affinity }(\mathrm{eV})\end{array}$ & LUMO (eV) \\
\hline \hline 1-Cl-naphthalene & 0.28 & -0.67 \\
1-Cl-anthracene & 0.75 & -1.07 \\
4-Br-nitrobenzene & 1.30 & -1.54 \\
\hline
\end{tabular}

rise to eq 2, which was used to estimate the electron affinities of the compounds of Figure 1:

$$
\mathrm{EA}_{\mathrm{X}}=-1.148(\mathrm{LUMO})_{\mathrm{X}}-0.478
$$

The resulting trend in electron affinity values (Table 2) is consistent with known values of similar compounds. It should be stressed that the calculated electron affinity values obtained are used only to generate a relative order of electron affinities for compounds 1-5 and are not meant to reflect absolute values. The relationship between analyte electron affinity and the extent of dehalogenation is illustrated in Figure 2. As can be seen in Figure 2, the extent of dehalogenation decreases with increasing analyte electron affinity. When the analyte electron affinity was high, as in the case of 5 , no dehalogenation was observed. However, it is relevant to wonder whether the electron affinity value of 5 corresponds to the electron affinity value at which dehalogenation tends toward zero. That limit may lie below the electron affinity value of 5 .

It has been proposed that radicals, electrons, ions, and excited species are produced in the matrix under FAB-LSIMS conditions. Of these species, radicals [19] and/or electrons [15] are thought to be involved in reduction processes. A dehalogenation mechanism has been proposed whereby kiloelectronvolt particle impacts cause the production of electrons (via ionization of the sample or matrix) and that these electrons can be effective reducing agents once they have reached thermal energies [15]. Subsequent capture of these beam-generated electrons by the analyte ultimately causes reductive dehalogenation (Scheme I). In the first instance, the secondary electron is captured by $\mathrm{Ar}-\mathrm{X}$, a haloaromatic species, to form the radical anion (reac- tion 3). This radical anion can decompose to expel the free halide (reaction 4). The resulting aromatic radical $\mathrm{Ar}^{-}$then abstracts a hydrogen from a solvent molecule to yield the dehalogenated aromatic species, ArH (reaction 5). This mechanism of reductive dehalogenation is in fact typical of the pulse radiolysis [20] and electrochemistry [21-24] literature.

$$
\begin{aligned}
& \mathrm{Ar}-\mathrm{X}+\mathrm{e} \longrightarrow \mathrm{Ar}^{-} \mathrm{X}^{-} \\
& \mathrm{Ar}-\mathrm{X}^{-} \longrightarrow \mathrm{Ar}+\mathrm{X}^{-} \\
& A r^{-}+\text {solvent } \longrightarrow \mathrm{ArH}
\end{aligned}
$$

Scheme I

On the basis of this mechanism, the inverse relationship of diminished dehalogenation with increasing analyte electron affinity appears to contradict the a priori notion that a molecule's propensity to dehalogenate should be commensurate with its ability to capture electrons. However, investigations carried out in pulse radiolysis [20] and electrochemistry [21-24] substantiate the trend observed in Figure 2. By using pulse radiolysis, Neta and Behar [20] studied the behavior of a series of substituted haloaromatics reduced by the solvated electron in irradiated aqueous solution. For a series of substituted halobenzenes with a given halogen, rates of dehalogenation were found to decrease as the electron affinity of the molecule increased. Similarly, extensive electrochemical investigations of reductive dehalogenation have upheld the validity of this relationship [21-24].

The inverse relationship of electron affinity and dehalogenation can be rationalized if the anion radical cleavage (reaction 4) is considered as an intramolecular dissociative electron transfer [22-24]. Originally, elec-

Table 2. Data used for the elaboration of Figure 2

\begin{tabular}{lrrr} 
Compound & \% Dehalogenation & LUMO $(\mathrm{eV})^{\mathrm{a}}$ & $\begin{array}{c}\text { Electron } \\
\text { affinity }(\mathrm{eV})^{\mathrm{b}}\end{array}$ \\
\hline $\mathbf{1}$ & 28 & -0.198 & -0.25 \\
2 & 24 & -0.329 & 0.10 \\
3 & 22 & -0.566 & 0.17 \\
4 & 13 & -0.852 & 0.50 \\
$\mathbf{5}$ & 0 & -1.299 & 1.01 \\
\hline a Estimated using MOPAC. & & & \\
${ }^{\mathrm{b}}$ Estimated using 2. & & &
\end{tabular}




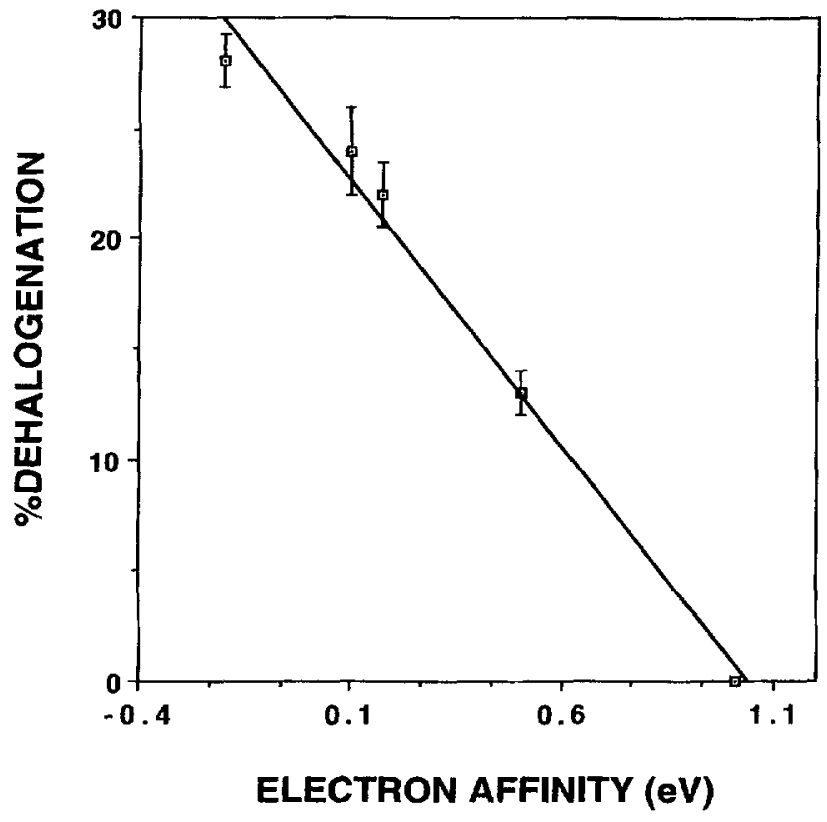

Figure 2. The effect of analyte electron affinity on the percent dehalogenation of the bromoaromatic compounds $1-5$. The equation of the linear relationship is $y=25.01-24.07 x(R=0.99)$. The error bars represent the standard deviation of three replicates.

tron capture occurs through the $\pi^{*}$ (LUMO) orbital. For dissociation of the radical anion to occur, the unpaired electron must be transferred from the $\pi^{*}$ (LUMO) orbital to the $\sigma^{*}$ orbital of the C-X bond. The likelihood of this intramolecular electron transfer depends on the energy gap between the two orbitals. The energy of the $\sigma^{*}$ orbital is not expected to be affected greatly by the nature of the aromatic moiety, whereas the $\pi^{*}$ MO is very much dependent on the nature of the aromatic moiety. In a series of aromatic compounds with the same halogen, the difference in energy between the $\sigma^{*}$ and the $\pi^{*}$ LUMO should increase concomitantly with an increase in electron affinity (lowering of LUMO energy). Hence, the greater the electron affinity, the greater the energy gap between the LUMO and the $\sigma^{*}$ orbital of the C-X bond, all of which results in a diminished propensity for the dissociative intramolecular electron transfer necessary for dehalogenation to occur. This statement holds for compounds capable of radical anions formation. However, given the negative electron affinity of compound 1 , dehalogenation in this case is more likely to proceed through dissociative electron capture (reaction 6):

$$
\mathrm{Ar}-\mathrm{X}+\mathrm{e} \longrightarrow \mathrm{Ar}^{*}+\mathrm{X}^{-}
$$

Interestingly, the trend illustrated in Figure 2 is in agreement with an electrochemical scale that determines haloarene radical anion stability. Reduction potentials generally follow the same trend as electron affinities [18, 25-28]. It has been suggested [29] that the reduction potential limit above which the haloarene radical anions can be considered stable is defined for chloro- and bromoaromatics. For chloroaromatics, the radical anions generated electrochemically will be stable if the reduction potential is more positive than $-1.6 \mathrm{~V}$ [29]. In the case of bromoaromatics, the reduction potential should be more positive than some value between -1.2 and $-1.6 \mathrm{~V}$ for the radical anions to be stable with respect to dehalogenation. The dehalogenation tendency of bromoaromatics should tend toward zero as the reduction potential of the compounds becomes more positive. In the case of the compounds used in this study, such as 4 and 5 , reduction potential values are unavailable. However, it is reasonable to assume that the reduction potentials of 4 and 5 can be roughly estimated from compounds that have similar structures. Thus, the reduction potential of the bromonitroitroaromatic compound 5 should be similar to that of 4-bromonitrobenzene $(-1.0 \mathrm{~V})$ and hence easily should fall above (more positive) the range of values $(-1.2$ to $-1.6 \mathrm{~V})$ where the radical anion will be considered stable. In accord with this reasoning, no dehalogenation is detected in the glycerol LSIMS spectrum of compound 5. The reduction potential of 4 should be similar to that of 4-bromoacetophenone $(-1.81 \mathrm{~V})$ [24]. Therefore, on the basis of the reduction potential we would expect the radical anion of 4 to be deemed unstable and dehalogenation to occur because its reduction potential is more negative than the range of values proposed $(-1.2$ to $-1.6 \mathrm{~V})$ where the radical anion will be considered stable. This is indeed the case: dehalogenation is observed in the glycerol LSIMS spectrum of compound 4. Of course, the criterion of anion radical stability is somewhat arbitrary, but the rough agreement of electrochemical studies [29] with our results is nonetheless interesting.

We have not established the same data base for chloroaromatics, but when the analyte electron affinity was high, no dehalogenation was observed as in the case of the bromoaromatic compound 5. For example, in the LSIMS spectrum of 4-(4-chlorobenzoyl)pyridine (compound 6) in glycerol, no dehalogenation was observed. The electron affinity of 6 can be estimated to be at least equal to that of 4 -Cl-benzophenone $(0.8 \mathrm{eV})$ [18] from the following argument. The reduction potential of 4-benzoylpyridine $(-1.51 \mathrm{~V}[30])$ is less negative than that of 4-Cl-benzophenone $(-1.6 \mathrm{~V})$ [24], which suggests that the electron affinity of 4-benzoylpyridine should be higher than that of 4-Cl-benzophenone. The chlorine substituent in the $4-$ position of 6 should increase its electron affinity relative to that of 4-benzoylpyridine, if it affects it at all. Thus, the radical anion formed by compound 6 is deemed to be stable according to the electrochemical scale that determines haloarene radical anion stability because its reduction potential should be more positive than $-1.6 \mathrm{~V}$ [29].

However, evidence of the reduction of compound 6 in the form of hydrogen addition ([M $\left.+n \mathrm{H}]^{+}, n>1\right)$ was indicated by the anomalous molecular ion region, which did not match the calculated isotopic contribu- 
tion from natural abundances. When a reduction-inhibiting matrix such as NBA was used, the isotopic pattern of the molecular ion region was closer to that of the calculated contribution of the natural isotopes. The effect of matrix selection on the molecular ion region is well illustrated in Figure 3 . One can suspect that the inhibition of the reduction process was only part ial because of the relatively high electron affinity of the analyte, which is close to that of the matrix $\left(\mathrm{EA}_{(\mathrm{NBA})}=0.9 \mathrm{eV}\right)[17]$.

For consideration of the possible involvement of species other than electrons in the beam-induced dehalogenation and/or reduction processes (as in the case of 6), it is useful to look at the reactive species generated upon kiloelectronvolt particle bombardment of a glycerol solution. For this purpose, it is appropriate to draw upon the radiation chemistry of neat alcohols to gain an understanding of the beam-induced radical cascade in glycerol. Although the energy regimes of most radiation chemistry techniques and FAB-LSIMS are different (megaelectronvolts versus kiloelectronvolts), a similarity lies in the way an energetic particle generates a track in the medium it penetrates, along which energy is deposited and radicals, ions, and electrons are formed. For example, upon irradiation of the simplest alcohol-methanol-a complicated mixture of reactive species is formed [31]:

$$
\mathrm{CH}_{3} \mathrm{OH} \sim \rightarrow \mathrm{e}^{-}, \mathrm{CH}_{2} \mathrm{OH}, \mathrm{CH}_{3} \mathrm{O}, \mathrm{H} ;{ }^{\circ} \mathrm{OH}_{r} \mathrm{CH}_{3}
$$

The reducing species (in order of "reducing power") are the electrons, hydrogen atoms, and $\alpha$-hydroxyalkyl radicals; the latter are carbon-centered radicals. The generation of such $\mathrm{C}$-centered radicals upon kiloelectronvolt particle bombardment of glycerol has been established firmly [19, 32-34]. These $\alpha$-hydroxyalkyl

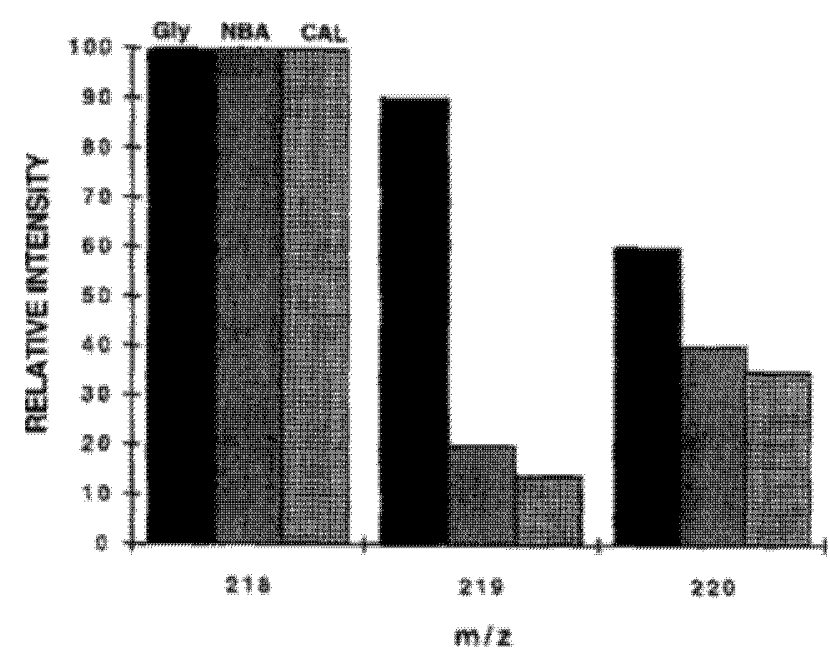

Figure 3. The molecular ion region of the LSIMS spectrum of 4-(4-Cl-benzoyl)pyridine (6) in glycerol (Gly) and nitrobenzyl alcohol (NBA). Also included is the natural isotopic distribution (CAL). radicals can be effective electron transfer agents [35]. It is reasonable to postulate that the electron transfer reactions of $\alpha$-hydroxyalkyl radicals can be used to estimate the likelihood of such reactions in FAB-LSIMS when glycerol is the matrix. The analysis of the beaminduced radical-radical coupling products that originate from neat glycerol indicates that one of the precursors to many such products is $\mathrm{HOCH}_{2} \mathrm{C} \cdot(\mathrm{OH}) \mathrm{CH}_{2} \mathrm{OH}$. This radical bears a structural resemblance to the $\alpha$-hydroxyisopropyl radical $\mathrm{CH}_{3} \mathrm{C}^{\prime}(\mathrm{OH}) \mathrm{CH}_{3}$, which can be generated from isopropanol in pulse radiolysis experiments. The results of a pulse radiolysis study indicate that the metalloporphyrin hemin IIIC is reduced by both radicals at comparable rates in aqueous solution [36].

The $\alpha$-hydroxyalkyl radicals generally can undergo efficient electron transfer with electron affinic aromatic compounds [35]. This is probably the case for compound 6 because pulse radiolysis experiments showed that the $\alpha$-hydroxyalkyl radical generated from isopropanol reduced 4-benzoylpyridine [37]. In a pulse radiolysis study of reductive dehalogenation, the authors concluded that $\alpha$-hydroxyalkyl radicals did not react with chorotoluene [38]. Our results indicate that dehalogenation does not occur when the electron affinity is high. Hence, it is reasonable to propose that $\alpha$-hydroxyalkyl radicals do not contribute significantly to the beam-induced dehalogenation process.

However, $\alpha$-hydroxyalkyl radicals possibly could be involved in the reduction of other compounds studied by FAB-LSIMS such as organic dyes. A pulse radiolysis study has demonstrated that these compounds undergo reduction through electron transfer with $\alpha$-hydroxyalkyl radicals generated from methanol and isopropanol [39]. Another possible example is the one electron reduction of the dicationic compound methyl viologen to yield $\mathrm{M}^{+\cdot}$ in the glycerol fast-atom bombardment (FAB) mass spectrum [40]. The involvement of $\alpha$-hydroxyalkyl radicals is confirmed by a pulse radiolysis investigation where it was established that methyl viologen was quickly reduced to $\mathrm{M}^{+*}$ by the $\mathrm{CH}_{2} \mathrm{OH}$ radical [41].

Where the contribution of hydrogen atoms to the dehalogenation process is concerned, pulse radiolysis experiments suggested that chloro- and bromobenzene do not react with hydrogen atoms to yield the free halide in aqueous solution [42]. The results of a $\gamma$-radiolysis study of octachlorobiphenyl in pure isopropanol indicated that the solvated electron was the primary species responsible for dechlorination. By a judicious choice of experimental conditions and scavenger types, the authors established that the hydrogen atom or $\alpha$-hydroxyalkyl radicals were not involved significantly in the dehalogenation process [43].

At this point, it is useful to attempt to define the reduction process undergone by compound 6 . However, the specific nature of the reduction process is difficult to define given the fact that $[\mathrm{M}+\mathrm{nH}]^{+}(n>1)$ species can arise from two pathways: (1) from electron 
capture followed by protonation or (2) direct attack of hydrogen radicals on the molecule. If the reduction of compound 6 through direct attack of hydrogen radicals is a significant reduction pathway, then the fact that no dehalogenation is observed strongly suggests that such radicals do not play an important role in the beam-induced dehalogenation of aromatic compounds. The capture of electrons to form a radical anion that can then undergo rapid protonation appears to fit the evidence at hand more elegantly.

The usefulness of the negative ion mode to monitor the dehalogenation process is limited. For example, it is reported that dehalogenation is reduced greatly in the negative FAB spectrum of 5-bromouridine compared to the dehalogenation observed in the positive FAB spectrum (28\% versus 9\%) [6]. A similar trend was observed in a later report [9]. Nevertheless, we have obtained the negative ion data for compounds 2 , 3,5 , and 6. The cationic compounds 1 and 4 do not give any useful signal in the negative ion mode. The spectrum obtained from 2 was too weak to be useful. The percent dehalogenation for 3 was $7 \%$ in the negative ion mode compared to $22 \%$ in the positive ion mode. As stated earlier, compound 5 does not undergo dehalogenation. The negative ion data for 3 underlines what is pointed out in the literature $[6,9]$ where the negative ion mode was used to monitor the dehalogenation process, namely, that the extent of dehalogenation observed in the nagative ion spectra is considerably lower (when not completely absent) than in the positive mode. Radical anions were observed for high electron affinity compounds such as $\mathbf{5}$ and $\mathbf{6}$. The mass spectrum of $\mathbf{6}$ is shown in Figure 4. The relative abundance due to the $\mathrm{Cl}^{-}$ion was negligible.

However, the explanation for the diminished extent of dehalogenation observed in the negative ion mode compared with the positive ion mode probably lies in the rapid extraction of the electrons in the negative mode. In the positive mode, the beam-generated electrons are retained and may actually be forced to diffuse into the bulk of the solution. Hence, although the

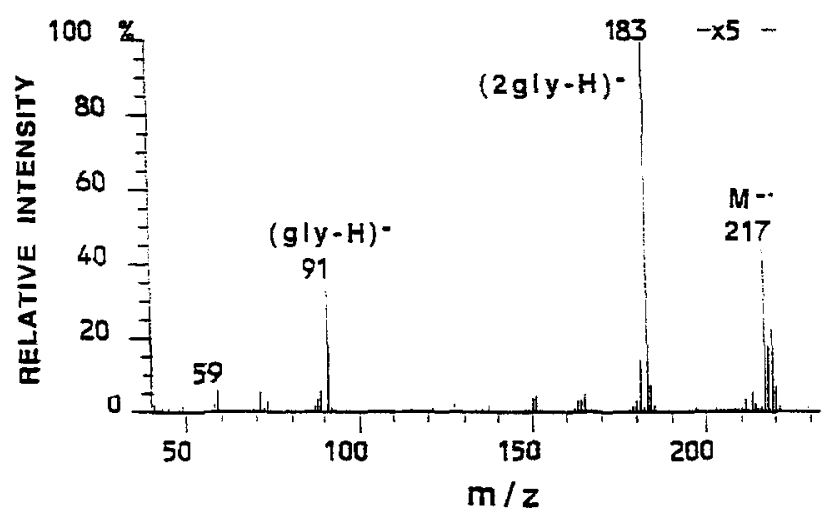

Figure 4. Negative ion LSIMS spectrum of 4-(4-Cl-benzoyl) pyridine (6) in glycerol. negative ion mode is not very useful in monitoring the effect of analyte electron affinity on the dehalogenation process, our negative ion data in conjunction with previous results $[6,9]$ add weight to an electron-based dehalogenation mechanism.

The observation that beam-induced dehalogenation decreases with increasing analyte electron affinity is consistent mechanistically with the direct involvement of secondary electrons in the dehalogenation process. This observation is consistent also with the observation that dehalogenation is quenched in matrices with high electron affinities [9-11]. Interestingly, a FAB study of the beam-induced reductive demetalation of metalloporphyrins showed that the incorporation of electron withdrawing groups in the porphyrin structure produced a significant decrease in reductive demetalation. The decrease in demetalation was explained in terms of slower electron transfer from the porphyrin moiety to the metal [44]. Whilst the occurrence of free radical reactions that lead to dehalogenation cannot be completely ruled out, the electron capture mechanism proposed here is more consistent with the data presented. The foregoing interpretation is supported by results obtained with other techniques, because a similar trend exists in pulse radiolysis [20] and electrochemistry [21-24] where reductive dehalogenation is initiated by the electron. These results substantiate the proposition that secondary electron production is an intrinsic part of the bombardment process.

\section{Conclusion}

The observation that beam-induced dehalogenation decreases with increasing analyte electron affinity is evidence that mechanistically is consistent with the proposition that secondary electron production is an intrinsic part of the bombardment process. Our results constitute evidence for the proposition made by others $[34,40,45-48]$ with regard to the initial processes that occur upon kiloelectronvolt particle impact where glycerol molecules are ionized through ejection of secondary electrons. The $\alpha$-hydroxyalkyl radicals that stem from the bombardment of glycerol are proposed to be potential contributors to beam-induced reduction processes because such radicals engage in one electron reductions. However, the contribution of $\alpha$-hydroxylalkyl radicals to the dehalogenation process does not appear to be significant. The usefulness of drawing upon the radiation chemistry literature to expand our current understanding of beam-induced reduction processes is demonstrated [11].

\section{Acknowledgments}

The assistance of Pierre-Nicolas Roy with MOPAC is gratefully acknowledged. The authors also acknowledge the financial contribution of the Natural Sciences and Engineering Research Council of Canada. 


\section{References}

1. Barber, M.; Bordoli, R. S.; Sedgewick, R. D.; Tyler, A. N. J. J. Chem. Soc. Chem. Commun. 1981, 325-327.

2. De Pauw, E. Adv. Mass Spectrom. 1981, 11, 383-401.

3. Detter, D. D.; Hand, O. W.; Cooks, R. G.; Walton, R. A. Mass Spectrom. Rev. 1988, 7, 465-503.

4. Schiebel, H. M.; Schulze, P.; Stohrer, W. D.; Leibfritz, D.; Jastorff, B.; Maurer, K. H. Biomed. Mass Spectrom. 1985, 12, $170-180$.

5. Dung, D. V.; Marien, J.; De Pauw, E.; Decuypr, J. Org. Mass Spectrom. 1984, 19, 276-279.

6. Sethi, S. K.; Nelson, C. C.; McCloskey, J. A. Anal. Chem. 1984, 56, $1975-1977$.

7. Nakaruma, T.; Nagaki, H.; Kinoshita, T. Bull. Chem. Soc. Japan. 1985, 58, 2798-2800.

8. Edom, R. W.; McKay, G.; Hubbard, J. W.; Midha, K. K. Biol. Mass Spectrom. 1991, 20, 585-592.

9. Kelley, J. A.; Musser, S. M. Org. Mass Spectrom. 1993, 28, 672-678

10. Théberge, R.; Paul, G.; Bertrand, M. J. Org. Mass Spectrom. 1994, 29, 18-25.

11. Théberge, R.; Bertrand, M. J.. J. Mass Spectrom. 1995, 1, $163-171$.

12. Yang, Y. M.; Fales, H. M.; Pannell, L. Anal. Chem. 1985, 57. $1771-1772$

13. Acheampong, A.; Nguyen, H.; Harcourt, D.; Tang-Liu, D.; Garst, M. Proceedings of the 41st Annual ASMS Conference on Mass Spectrometry and Allied Topics; Washington, DC, 1993; pp $48 \mathrm{a}-48 \mathrm{~b}$.

14. Volmer, D.; Levsen, K. J. Am. Soc. Mass Spectrom. 1994, 5 , 655-675.

15. Williams, D. H.; Findeis, A. F.; Naylor, S.; Gibson, B. W. J. Am. Chem. Soc. 1987, 109, 1980-1986.

16. Klausner, Y. S.; Bodansky, M. Synthese 1972, 453-463.

17. Laramée, J. A.; Arbogast, B.; Deinzer, M. L. Anal. Chem. 1989, 67, 171-175.

18. Wentworth, W. E.; Chen, E. C. M. Cryst. Liq. Cryst. 1989, 171, 270-285.

19. Field, F. H. J. Phys. Chem. 1982, 86, 5115-5123.

20. Neta, P; Behar, D. J. Am. Chem. Soc. 1981, 103, 2280-2283.

21. Andrieux, C. P.; Savéant, J. M.; Zann, D. Nouvo J. Chim. 1984, $8,107-116$.

22. Savéant, J. M. Adv. Phys. Org. Chem. 1990, 26, 1-130.
23. Rossi, R. A. Acc. Chem. Res. 1982, 15, 164-170.

24. Savéant, J. M. Bull. Soc. Chim. Fr. 1988, 225-237.

25. Kebarle, P.; Chowdhury, S. Chem. Rev. 1987, 87, 513-534.

26. Paul, G.; Kebarle, P. I. Am. Chem. Soc. 1989, 111, 464-470.

27. Tanner, D. D.; Deonarian, N.; Kharrat, A. Can. J. Chem. 1989, 67, 171-175.

28. Shalev, H.; Evans, D. H. I. Am. Chem. Soc. 1989, 111, $2667-2674$

29. Alwair, K.; Grimshaw, J. J. Chem. Soc. Perkin Trans. 2 1973, 1811-1815.

30. Ching-Fong Shu; Wrighton, M. S. Inorg. Chem. 1988, 27, 4326-4329.

31. Bensasson, R. V.; Land, E. J.; Truscott, T. G. Flash Photolysis and Pulse Radiolysis: Contributions to the Chemistry and Biology of Medicine; Pergamon: New York, 1983; pp 8-9.

32. Keough, T.; Ezra, F. S.; Russell, A. F.; Pryne, J. D. Org. Mass Spectrom. 1987, 22, 241-247.

33. Tuinman, A. A.; Cook, K. D. I. Am. Soc. Mass Spectrom 1994, $5,92-97$.

34. Caldwell, K. A.; Gross, M. L. J. Am. Soc. Mass Spectrom. 1994, $5,72-91$.

35. Swallow, A. G. Prog. React. Kinet. 1978, 9, 195-366.

36. Goff, H.; Simic, M. G. Biochim. Biophys. Acta 1975, 392, 201-207.

37. Nelson, D. A.; Hayon, E. J. Phys. Chem. 1972, 76, 3200-3207.

38. Mohan, H.; Mudaliar, M.; Rao, B. S. M.; Mittal, J. P. Radiat. Phys. Chem. 1992, 40, 513-517.

39. Rao, P. S.; Hayon, E. J. Phys. Chem. 1973, 77, 2753-2756.

40. Clayton, E.; Wakefield, A. J. C. I. Chem. Soc. Chem. Commun. 1984, 969-970.

41. Patterson, L. K.; Small, R. D.; Scaiano, J. C. Radiat. Res. 1977, $72,218-225$.

42. Lichtscheidl, J.; Getoff, N. Monatsh. Chem. 1979, 110, 1377-1381.

43. Mincher, B. J.; Arbon, R. E.; Knighton, W. B.; Meikrantz, D. H. Appl. Radiat. Isot. 1994, 45, 879-887.

44. Naylor, S.; Hunter, C. A.; Cowan, J. A.; Lamb, J. H.; Sanders, J. K. M. J. Am. Chem. Soc. 1990, 112, 6507-6514.

45. Sunner, J. Org. Mass Spectrom. 1993, 28, 805-824.

46. Katz, R. N.; Field, F. H. Proceedings of the 11th International Mass Spectrometry Conference; Bordeaux, 1988; pp 1022-1023.

47. Sunner, J.; Kulatunga, R.; Kebarle, P. Anal. Chem. 1986, 58, 2009-2014.

48. Ligon, W. V. Int. J. Mass Spectrom. Ion Phys. 1983, 52, 189-191. 